\section{Cirugías más frecuentes en el Centro Médico Naval en un período de 5 años}

\section{Most frequent surgeries at the Naval Medical Center in a 5-year period}

\author{
LE Adriana Esther Díaz-Medina,* Dr. Ángel Gabriel Vargas-Ruiz, ${ }^{\ddagger}$ \\ Dr. Gabriel Lara-Hernández ${ }^{\S}$
}

Citar como: Díaz-Medina AE, Vargas-Ruiz ÁG, Lara-Hernández G. Cirugías más frecuentes en el Centro Médico Naval en un período de 5 años. Rev Mex Anestesiol. 2021; 44 (3): 158-165. https://dx.doi.org/10.35366/99661

\begin{abstract}
RESUMEN. Introducción: El objetivo de este estudio es determinar los procedimientos quirúrgicos más frecuentemente realizados en el Centro Médico Naval, un Centro Médico de Tercer Nivel. Material y métodos: Se realizó un estudio observacional, exploratorio, descriptivo, trasversal y retrospectivo incluyendo todas las cirugías realizadas del 01 de enero de 2015 al 31 de julio de 2019, consultando los registros del quirófano y los expedientes electrónicos. Resultados: Se registraron 25,114 cirugías, con un promedio anual de 5,527 procedimientos y de 16 procedimientos diarios (rango de 2 a 25). Las especialidades que más cirugías realizaron fueron cirugía general, ortopedia, ginecoobstetricia y oftalmología. Las principales cirugías realizadas fueron la facoemulsificación de catarata con colocación de lente intraocular, endoscopías, cesáreas, colecistectomía laparoscópica, atención del trabajo de parto, reducción abierta con fijación interna de fracturas, apendicectomía abierta, hernioplastía inguinal y lavados quirúrgicos. El 59\% de las cirugías se realizó en mujeres. El rango de edad de los pacientes fue desde recién nacido hasta los 101 años, estando la mayoría entre los 53 y 72 años. Conclusiones: El Centro Médico Naval destina la mayoría de los recursos de su quirófano para la atención obstétrica (cesárea y trabajo de parto), la atención de padecimientos crónico-degenerativos (cirugía de catarata, y endoscopías), atención de accidentes (reducción de fracturas y lavados quirúrgicos) y la resolución de padecimientos abdominales agudos como la apendicitis y la colecistitis.
\end{abstract}

ABSTRACT. Introduction: The study objective was to determine the most frequently surgical procedures at the Naval Medical Center, a third level medical center. Material and methods: An observational, exploratory, descriptive, cross-sectional and retrospective study was conducted including all surgeries performed from January 1, 2015 to July 31, 2019, reviewing the surgical records and patient electronic files. Results: 25,114 surgeries were registered, with an annual average of 5,527 procedures and 16 daily procedures (range from 2 to 25). General surgery, orthopedics, gynecoobstetrics and ophthalmology were the specialties with the most amount of surgeries. The main surgeries were cataract phacoemulsification with intraocular lens placement, endoscopies, caesarean sections, laparoscopic cholecystectomy, labor delivery, open reduction with internal fracture fixation, open appendectomy, inguinal hernioplasty and surgical washes. 59\% of the surgeries were performed in women. The age range of the patients was from newborn to 101 years, with most patients between 53 and 72 years. Conclusions: The naval medical center spend surgical resources for obstetric care (caesarean section and labor), care of chronic degenerative diseases (cataract surgery, and endoscopy), accident care (fracture reduction and surgical washes) and the resolution of acute abdominal conditions such as appendicitis and cholecystitis.

\section{INTRODUCCIÓN}

L a cirugía se encuentra dentro de las especialidades troncales de la atención médica. En los hospitales de tercer nivel de atención médica, el Servicio de Cirugía se encuentra dentro de los servicios prioritarios necesarios para brindar una adecuada atención médica. Con el tiempo, las intervenciones quirúrgicas se han vuelto cada vez más complejas y especializadas, lo que exige que los servicios quirúrgicos y los quirófanos se encuentren en constante renovación, además de que, al crecer cada vez más la población derechohabiente, existe una demanda creciente del número de cirugías ${ }^{(1)}$.

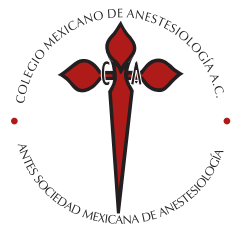

Palabras clave: Cirugía, procedimiento quirúrgico, quirófano, cirujano.

Keywords:

Surgery, surgical procedure, operating room, surgeon.

* Licenciado en Enfermería. Postgrado en Enfermería Quirúrgica.

${ }^{\ddagger}$ Médico Especialista en Medicina Interna y Hematología.

$\S$ Coordinador de Investigación «A», Escuela de Postgrado en Sanidad Naval.

Centro Médico Naval. Secretaría de Marina Armada de México.

Correspondencia:

Adriana Esther Díaz-Medina

Avenida Heroica Escuela

Naval Militar Núm. 745, Col: Presidentes Ejidales 1ra. Sección, 04470, Alcaldía Coyoacán, CDMX E-mail: ady_184@hotmail.com

Recibido: 08-01-2020

Aceptado:13-03-2020

Abreviaturas:

$\mathrm{AV}=$ Arteriovenoso.

CEMENAV = Centro Médico Naval. HIS = Sistema Informático

Hospitalario.

HOSGENAES = Hospital General

Naval de Alta Especialidad.

IMSS = Instituto Mexicano

del Seguro Social.

MO = Médula ósea.

OMS = Organización

Mundial de la Salud. 
Con el avance del conocimiento en todas las ramas de la medicina, incluyendo la cirugía, las técnicas quirúrgicas exigen cada vez más especialización de los cirujanos, anestesiólogos y del personal de enfermería que labora en el quirófano ${ }^{(2)}$.

Conocer el número y las características de las cirugías realizadas en los quirófanos permite a la directiva del hospital tener una adecuada planeación de los recursos físicos, económicos y humanos para hacer que los quirófanos tengan una mayor productividad y calidad en su atención ${ }^{(3)}$. Además, conocer cuáles son las cirugías más realizadas permitirá que los profesores titulares de los cursos de especialización en cirugía general y en enfermería quirúrgica puedan adecuar los programas de educación quirúrgica, de acuerdo con las necesidades reales de capacitación en el quirófano.

En la actualidad, la Secretaría de Marina-Armada de México cuenta a nivel nacional con un Centro Médico Naval, 13 hospitales, 10 clínicas y 13 sanatorios navales, que proporcionan servicios médicos a una población de 283,434 elementos, además de secciones sanitarias en Unidades operativas que requieren de al menos un médico general y un enfermero.

El presente estudio tiene como finalidad determinar las cirugías más frecuentes que se han realizado en el quirófano del Centro Médico Naval durante los últimos cinco años (2015-2019). El Centro Médico Naval (CEMENAV), antes conocido como Hospital General Naval de Alta Especialidad (HOSGENAES), es un hospital de tercer nivel creado el 01 de noviembre del 2008 con la finalidad de brindar atención médica integral especializada al personal militar naval y a sus derechohabientes (Ficha logística del Hospital General Naval de Alta especialidad, 2011).

\section{MATERIAL Y MÉTODOS}

Se realizó un estudio observacional, exploratorio, descriptivo, trasversal y retrospectivo con la finalidad de determinar cuáles son los procedimientos quirúrgicos que más frecuentemente

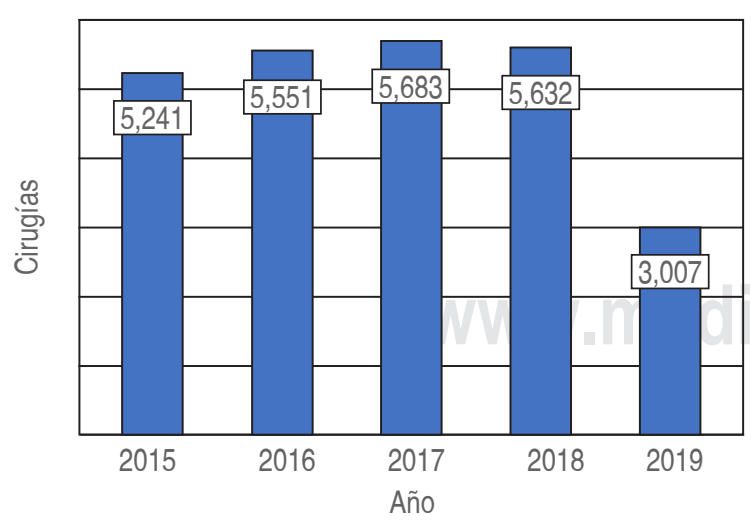

Figura 1: Número de cirugías realizadas en el CEMENAV de enero de 2015 a julio 2019 divididas por año.

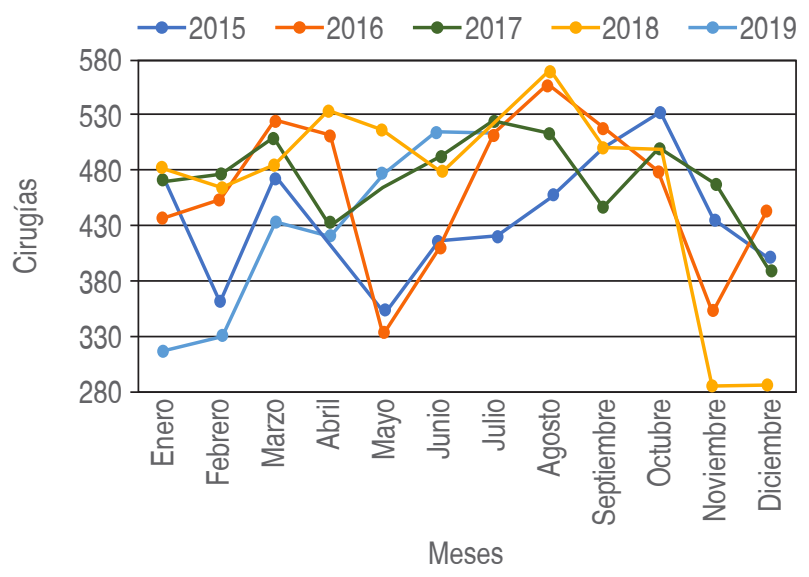

Figura 2: Número de cirugías realizadas en el CEMENAV de enero 2015 a julio 2019 agrupadas de acuerdo con los meses del año.

se realizan en el CEMENAV, describir a qué especialidad quirúrgica pertenecen dichas cirugías, los tipos de cirugía más comunes realizados por género, determinar el promedio de procedimientos quirúrgicos por mes y por año realizados en el quirófano del Centro Médico Naval y el rango de edad de los pacientes atendidos en el quirófano.

El estudio contó con la aprobación del Comité de Ética del CEMENAV y se apegó a las normas y líneas de investigación de la Universidad Naval.

Se incluyeron todos los procedimientos realizados en el quirófano en un período del 01 de enero de 2015 al 31 de julio de 2019, recogiendo los datos de los registros de la programación diaria de cirugías en el quirófano y del sistema de información hospitalaria (HIS).

Los datos recabados se analizaron usando estadística descriptiva, como medidas de tendencia central y se representaron usando proporciones y porcentajes de frecuencias en cuadros y figuras. Se utilizó el software estadístico SPSS versión 25.

\section{RESULTADOS}

\section{Número de procedimientos quirúrgicos realizados en el CEMENAV}

Del 01 de enero de 2015 al 31 de julio de 2019 se registraron un total de 25,114 procedimientos quirúrgicos realizados en los quirófanos del Centro Médico Naval.

\section{Promedio de procedimientos quirúrgicos por año, mes y día en los quirófanos del CEMENAV}

Durante el período de tiempo comprendido por el estudio (01 de enero de 2015 a 31 de julio de 2019), el año en el que se realizaron un mayor número de cirugías fue 2017, con un total 


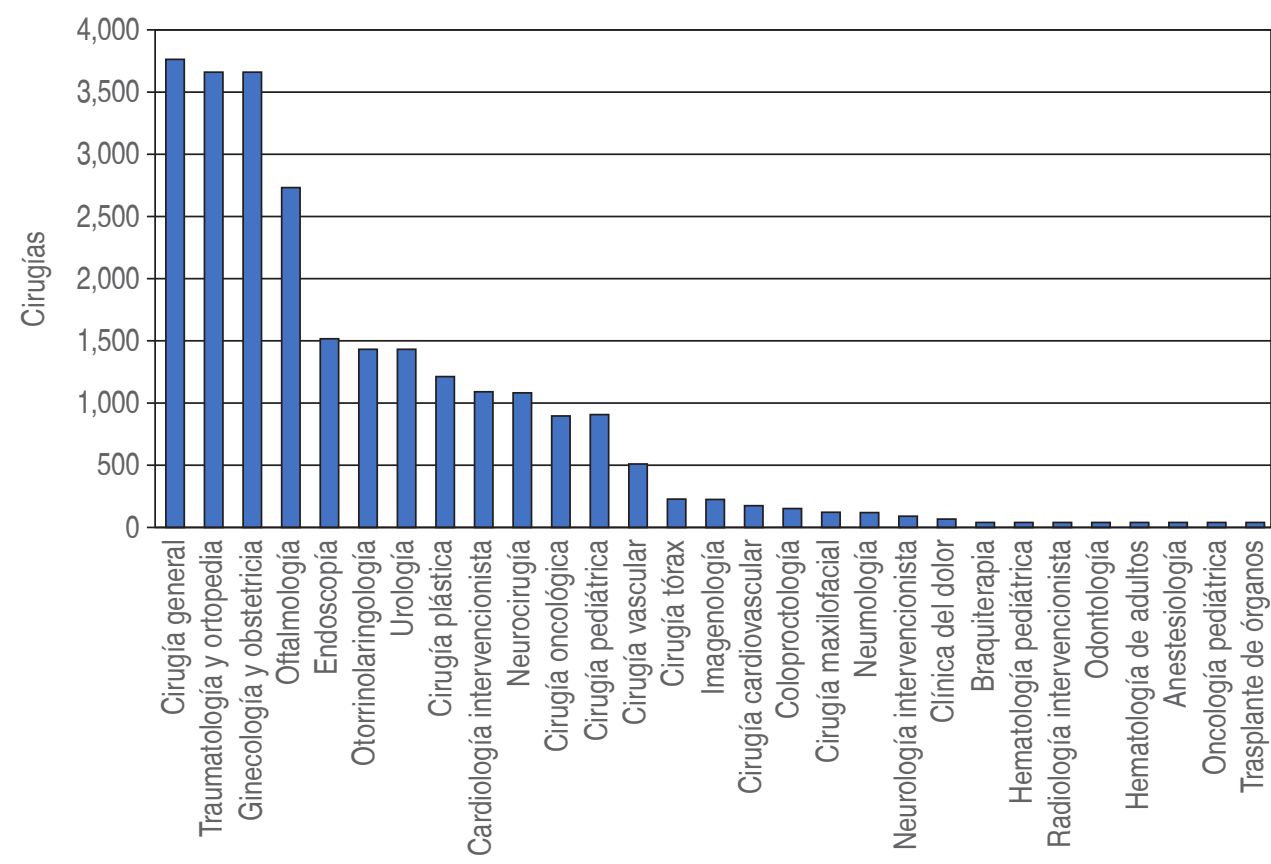

Especialidades

de 5,683 cirugías, y el año en el que menos procedimientos se registraron fue 2019 con 3,007 cirugías debido a que sólo se registraron los procedimientos de enero a julio de 2019 (Figura 1). El promedio de cirugías anuales fue de 5,527 procedimientos. La mediana de cirugías diarias fue de 16 procedimientos, con un rango de 2-25, siendo los fines de semana los días con un menor número de procedimientos realizados. La mediana de cirugías mensuales fue de 437, con un rango de 287 a 573 procedimientos mensuales.

El número de cirugías mensuales es muy parecido a lo largo de todos los meses del año, aunque es claro que durante los meses de mayo y diciembre el número de procedimientos fue más bajo y durante los meses de agosto y septiembre más alto. El comportamiento de acuerdo con los meses del año puede observarse en la Figura 2.

\section{Especialidades que realizan el mayor número de procedimientos}

Durante el período de estudio, 29 especialidades médicoquirúrgicas hicieron uso de los quirófanos del CEMENAV.

Las especialidades con mayor número de cirugías realizadas fueron cirugía general, traumatología y ortopedia, ginecología y obstetricia, oftalmología, endoscopía, otorrinolaringología, urología, cirugía plástica, cardiología intervencionista y neurocirugía. Por el contrario, las especialidades con el menor número de procedimientos fueron trasplante de órganos, oncología pediátrica, anestesiología, hematología de adultos y odontología. Esto se puede observar en la Figura 3.

\section{Procedimientos quirúrgicos más frecuentes}

Del 01 de enero de 2015 al 31 de julio de 2019 en los quirófanos del CEMENAV se realizaron 629 diferentes tipos de procedimientos quirúrgicos, los 10 procedimientos más fre-

\begin{tabular}{|c|c|c|}
\hline \multicolumn{3}{|c|}{$\begin{array}{l}\text { Tabla 1: Los } 20 \text { procedimientos quirúrgicos más } \\
\text { realizados en el CEMENAV de enero } 2015 \text { a julio } 2019 .\end{array}$} \\
\hline & Procedimiento quirúrgico & n (\%) \\
\hline 1 & $\begin{array}{l}\text { Facoemulsificación de catarata y colocación de } \\
\text { lente intraocular }\end{array}$ & $1,269(5.1)$ \\
\hline 2 & Endoscopía & $1,041(4.1)$ \\
\hline 3 & Cesárea Kerr & $754(3.0)$ \\
\hline 4 & Colecistectomía laparoscópica & $691(2.8)$ \\
\hline 5 & Atención del trabajo de parto & $664(2.6)$ \\
\hline 6 & Reducción abierta y fijación interna de fracturas & $607(2.4)$ \\
\hline 7 & Apendicectomía abierta & $520(2.1)$ \\
\hline 8 & Hernioplastía inguinal & $480(1.9)$ \\
\hline 9 & Cesárea con OTB & $452(1.8)$ \\
\hline 10 & Lavado quirúrgico & $412(1.6)$ \\
\hline 11 & Histerectomía & $399(1.6)$ \\
\hline 12 & Endoscopía y colonoscopía & $374(1.5)$ \\
\hline 13 & Aspiración manual endouterina & $350(1.4)$ \\
\hline 14 & Laparotomía exploradora & $344(1.4)$ \\
\hline 15 & Aplicación de anti angiogénico intravítreo & $319(1.3)$ \\
\hline 16 & Resección transuretral de próstata & $303(1.2)$ \\
\hline 17 & Artroplastía total de rodilla & $297(1.2)$ \\
\hline 18 & Rinoseptumplastía con turbinoplastía & $297(1.2)$ \\
\hline 19 & Angioplastía coronaria & $294(1.2)$ \\
\hline 20 & Colonoscopía & $268(1.1)$ \\
\hline
\end{tabular}

CEMENAV $=$ Centro Médico Naval, OTB = oclusión tubaria bilateral 
Tabla 2: Detalle de los procedimientos quirúrgicos más frecuentes realizados en el CEMENAV de enero 2015 a julio 2019 divididos por especialidades.

\begin{tabular}{|c|c|c|}
\hline Especialidad & Procedimientos quirúrgicos & $\mathrm{n}(\%)$ \\
\hline Anestesiología & Parche hemático & $5(100.0)$ \\
\hline Braquiterapia & Braquiterapia intracavitaria & $41(100.0)$ \\
\hline \multirow[t]{3}{*}{ Cardiología intervencionista } & Angioplastía coronaria & $285(26.4)$ \\
\hline & Coronariografía diagnóstica & $199(18.4)$ \\
\hline & Estudio electrofisiológico con ablación & $138(12.8)$ \\
\hline \multirow[t]{3}{*}{ Cirugía de tórax } & Simpatectomía torácica & $37(16.1)$ \\
\hline & Biopsia pulmonar & $28(12.2)$ \\
\hline & Lobectomía & $27(11.7)$ \\
\hline \multirow[t]{3}{*}{ Cirugía cardiovascular } & Revascularización coronaria & $36(23.7)$ \\
\hline & Ventana pericárdica & $17(11.2)$ \\
\hline & Cambio valvular aórtico por prótesis biológica & $15(9.9)$ \\
\hline \multirow[t]{3}{*}{ Cirugía general } & Colecistectomía laparoscópica & $683(18.5)$ \\
\hline & Hernioplastía inguinal & $430(11.6)$ \\
\hline & Apendiciectomía abierta & $420(11.4)$ \\
\hline \multirow[t]{3}{*}{ Cirugía maxilofacial } & Osteotomía Le Fort & $39(31.7)$ \\
\hline & Reducción de fractura maxilar & $22(17.9)$ \\
\hline & Palatofaringoplastía & $14(11.4)$ \\
\hline \multirow[t]{3}{*}{ Cirugía pediátrica } & Apendiciectomía abierta & $97(10.8)$ \\
\hline & Orquidopexia & $83(9.2)$ \\
\hline & Circuncisión & $71(7.9)$ \\
\hline \multirow[t]{3}{*}{ Cirugía plástica } & Reconstrucción de mama & $111(9.0)$ \\
\hline & Lavado quirúrgico & $107(8.7)$ \\
\hline & Liberación del túnel del carpo & $76(6.2)$ \\
\hline \multirow[t]{3}{*}{ Cirugía vascular } & Safenoablación & $216(42.2)$ \\
\hline & Construcción de fistula arteriovenosa para hemodiálisis & $56(10.9)$ \\
\hline & Amputación de miembro pélvico & $37(7.2)$ \\
\hline \multirow[t]{3}{*}{ Cirugía oncológica } & Mastectomía & $168(18.5)$ \\
\hline & Laparotomía exploradora citorreductora & $124(13.7)$ \\
\hline & Biopsia de mama guiada por imagen & $83(9.2)$ \\
\hline \multirow[t]{3}{*}{ Clínica del dolor } & Depósito de esteroide peridural & $17(26.6)$ \\
\hline & Colocación de bomba de infusión intratecal & $8(12.5)$ \\
\hline & Radiofrecuencia de rama media & $8(12.5)$ \\
\hline \multirow{3}{*}{ Coloproctología } & Fistulotomía anal & $73(52.1)$ \\
\hline & Hemorroidectomía & $36(25.7)$ \\
\hline & Colonoscopía & $5(3.6)$ \\
\hline \multirow[t]{3}{*}{ Endoscopía } & Endoscopía & $877(57.7)$ \\
\hline & Endoscopía y colonoscopía & $342(22.5)$ \\
\hline & Colonoscopía & $214(22.5)$ \\
\hline \multirow[t]{3}{*}{ Ginecología y obstetricia } & Cesárea Kerr & $752(20.6)$ \\
\hline & Atención del trabajo de parto & $664(18.2)$ \\
\hline & Cesárea con OTB & $451(12.3)$ \\
\hline Neumología & Broncoscopía & $90(85.7)$ \\
\hline \multirow[t]{2}{*}{ Hematología pediátrica } & Quimioterapia intratecal & $17(63.0)$ \\
\hline & Aspirado de MO y biopsia de hueso & $8(29.6)$ \\
\hline Hematología de adultos & Aspirado de MO y biopsia de hueso & $4(57.1)$ \\
\hline \multirow[t]{3}{*}{ Imagenología } & Sedación para resonancia & $149(65.4)$ \\
\hline & Sedación para tomografía & $57(25.0)$ \\
\hline & Sedación para PET-CT & $2(0.9)$ \\
\hline \multirow{3}{*}{ Neurocirugía } & Fusión lumbar transforaminal & $167(15.9)$ \\
\hline & Discectomía cervical anterior con fusión & $160(15.3)$ \\
\hline & Discectomía & $90(8.6)$ \\
\hline \multirow[t]{3}{*}{ Neurología intervencionista } & Angiografía cerebral con embolización de aneurisma & $34(37.0)$ \\
\hline & Angiografía cerebral & $25(27.2)$ \\
\hline & Angiografía carotidea con angioplastía & $9(9.8)$ \\
\hline \multirow[t]{2}{*}{ Odontología } & Rehabilitación bucal bajo anestesia & $5(62.5)$ \\
\hline & Odontectomía & $3(37.5)$ \\
\hline Oftalmología & Facoemulsificación de catarata y colocación de lente intraocular & $1,219(37.5)$ \\
\hline & Aplicación de antiangiogénico intravítreo & $319(11.7)$ \\
\hline
\end{tabular}




\begin{tabular}{lcc}
\hline & $\begin{array}{c}\text { Continúa la Tabla 2: Detalle de los procedimientos quirúrgicos más frecuentes realizados } \\
\text { en el CEMENAV de enero 2015 a julio 2019 divididos por especialidades. }\end{array}$ & $\mathrm{n}(\%)$ \\
\hline Especialidad & Procedimientos quirúrgicos & $158(11.7)$ \\
\hline & Extracción de catarata y colocación de lente intraocular & $297(20.6)$ \\
Otorrinolaringología & Rinoseptumplastía con turbinoplastía & $169(11.7)$ \\
& Rinoseptumplastía & $148(10.3)$ \\
Traumatología y ortopedia & Adenoamigdalectomía & $599(16.4)$ \\
& Reducción abierta y fijación interna de fracturas & $289(7.9)$ \\
Urología & Artroplastía total de rodilla & $253(6.9)$ \\
& Retiro de material de osteosíntesis & $303(6.9)$ \\
Radiología intervencionista & Resección transuretral de próstata & $175(12.2)$ \\
& Ureterolitotricia & $79(5.5)$ \\
& Prostatectomía radical & $4(30.8)$ \\
Oncología pediátrica & Biopsia renal & $2(15.4)$ \\
Trasplante de órganos & Nefrostomía & $2(15.4)$ \\
\hline
\end{tabular}

CEMENAV = Centro Médico Naval, OTB = Oclusión tubaria bilateral, MO = Médula ósea, PET-CT = Tomografía computada por emisión de positrones

cuentes fueron: facoemulsificación de catarata con colocación de lente intraocular, endoscopía, cesárea Kerr, colecistectomía laparoscópica, atención del trabajo de parto, reducción abierta y fijación interna de fracturas, apendicectomía abierta, hernioplastía inguinal, cesárea con oclusión tubaria bilateral (OTB) y el lavado quirúrgico. Las principales cirugías se muestran en la Tabla 1.

\section{Cirugías más frecuentes de cada especialidad}

El detalle de los procedimientos más comunes divididos por especialidad se encuentra en la Tabla 2.

\section{Procedimientos quirúrgicos más comunes de acuerdo con el género}

De acuerdo con el género, hubo más cirugías realizadas en mujeres: 10,280 cirugías se realizaron en hombres (40.9\%) y 14,843 cirugías se realizaron en mujeres (59.1\%). Al excluir las intervenciones obstétricas (como las cesáreas y la atención del trabajo de parto), el porcentaje de mujeres se reduce de 59.1 a $55.8 \%$, persistiendo el predominio del género femenino.

En los hombres (Tabla 3), la cirugía más común fue la facoemulsificación de catarata con colocación de lente intraocular seguido de la endoscopía, la hernioplastía inguinal, las reducciones abiertas con fijación interna de fracturas y la resección transuretral de próstata. En las mujeres (Tabla 4), la cirugía más frecuente fue la cesárea Kerr, seguida de la facoemulsificación de catarata con colocación de lente intraocular, la atención del trabajo de parto, la endoscopía y la colecistectomía laparoscópica.
En menores de 18 años el 55.4\% fueron hombres y el $44.6 \%$ mujeres. En el grupo de edad comprendido entre los 18 y 64 años hubo más pacientes mujeres (67\%) que hombres (33\%). En mayores de 65 años nuevamente vuelve a haber una proporción similar de hombres y de mujeres (52 y 48\% respectivamente), esto puede observarse en la Figura 4.

\section{Procedimientos quirúrgicos de acuerdo con la edad}

Respecto a la edad, el rango fue desde recién nacidos hasta los 101 años, con una distribución bimodal en la frecuencia de cirugías por edad (como se puede observar en la Figura 5), con el pico más cuantioso de procedimientos en pacientes

\begin{tabular}{rlr}
\multicolumn{3}{c}{ Tabla 3: Los diez principales procedimientos } \\
quirúrgicos más frecuentes en hombres en el CEMENAV \\
durante el período de enero 2015 a julio 2019.
\end{tabular}

CEMENAV = Centro Médico Naval. 
de 53 a 72 años, y un segundo pico más pequeño de los 28 a 36 años. El 10.7\% de los pacientes fueron menores de 18 años y el 25.7\% de los pacientes tuvieron 65 años o más. En los menores de edad, los principales procedimientos fueron los relacionados con sedación para estudios de imagen como resonancia o tomografía, adenoamigdalectomía, epifisiodesis (correctora de algunos defectos óseos congénitos) y apendicectomía. En los pacientes más jóvenes predominan las intervenciones obstétricas (cesárea con o sin OTB, atención del trabajo de parto, aspiración manual endouterina, etcétera) y a medida que la edad de los pacientes aumenta, aparecen otros procedimientos quirúrgicos como la colecistectomía, endoscopía o histerectomía, mientras que en la población mayor de 65 años predominan cirugías que se relacionan a padecimientos crónico-degenerativos como la cirugía de catarata o las artroplastías de rodilla, y a los problemas neoplásicos como la prostatectomía y la mastectomía.

\section{DISCUSIÓN}

La finalidad de este trabajo de investigación es dar a conocer el panorama de las principales intervenciones quirúrgicas y otros procedimientos realizados en los quirófanos del Centro Médico Naval.

En el CEMENAV se realizan un promedio de 5,527 cirugías al año y 16 procedimientos quirúrgicos en un día promedio. Aunque el número de cirugías está distribuido de manera uniforme a lo largo del año, los meses de mayo y diciembre tienden a tener el número más bajo de cirugías, probablemente en relación con los períodos vacacionales más grandes que tiene el año.

El 60\% de las cirugías se realiza en mujeres, en quienes las principales cirugías se relacionan con la atención

\begin{tabular}{|c|c|c|}
\hline & Procedimiento & n (\%) \\
\hline 1 & Cesárea Kerr & $749(5.0)$ \\
\hline 2 & $\begin{array}{l}\text { Facoemulsificación de catarata y colocación de } \\
\text { lente intraocular }\end{array}$ & $715(4.8)$ \\
\hline 3 & Atención del trabajo de parto & $660(4.4)$ \\
\hline 4 & Endoscopía & $627(4.2)$ \\
\hline 5 & Colecistectomía laparoscópica & $497(3.4)$ \\
\hline 6 & Cesárea con OTB & $450(3.0)$ \\
\hline 7 & Histerectomía & $397(2.7)$ \\
\hline 8 & Aspiración manual endouterina & $344(2.3)$ \\
\hline 9 & Apendiciectomía abierta & $270(1.8)$ \\
\hline 10 & Endoscopía y colonoscopía & $245(1.7)$ \\
\hline
\end{tabular}

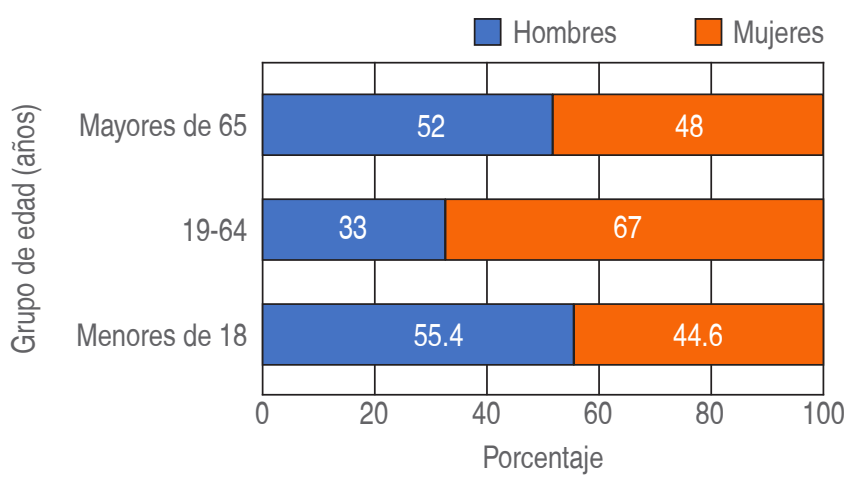

Figura 4: Distribución de los pacientes por género de acuerdo con los grupos de edad.

obstétrica, como son la cesárea (con o sin OTB), atención del trabajo de parto, histerectomía y aspiración manual endouterina. El 40\% de los procedimientos se realizó en hombres. En ambos géneros, cirugías como faciemulsificación de catarata con colocación de lente intraocular o las apendicectomías fueron frecuentes, aunque en las mujeres las colecistectomías laparoscópicas fueron tres veces más frecuentes en comparación con los hombres (497 vs 194 casos). En la lista de procedimientos más frecuentes en hombres aparecen las reducciones de fracturas, la prostatectomía transuretral y la angioplastía coronaria, que no aparecen en la lista de procedimientos más frecuentes en mujeres y que refleja patologías propias del género masculino (como la prostatectomía) o patologías que ya se conoce son más frecuentes en hombres que en mujeres (como la cardiopatía isquémica y los accidentes).

En el quirófano, se atendieron pacientes de todas las edades, desde recién nacidos hasta los 101 años. Sin embargo, es clara la distribución bimodal de la edad, con el pico más grande de procedimientos en personas de 53 a 72 años y otro más pequeño en jóvenes, de 28 a 26 años, en el cual las mujeres predominan. Los procedimientos también van cambiando con la edad, así en los menores de edad, los principales procedimientos fueron los relacionados con sedación para estudios de imagen como resonancia o tomografía, adenoamigdalectomía, epifisiodesis (correctora de algunos defectos óseos congénitos) y apendiciectomía. En los pacientes más jóvenes predominaron las intervenciones obstétricas (cesárea con o sin OTB, atención del trabajo de parto, aspiración manual endouterina, etcétera) ya que la mayoría fueron mujeres y a medida que la edad aumenta aparecen otros procedimientos quirúrgicos como la colecistectomía, endoscopía o histerectomía, mientras que en la población mayor de 65 años predominan cirugías que se relacionan a padecimientos crónico-degenerativos como la cirugía de catarata o las artroplastías de rodilla y a los problemas neoplásicos como la prostatectomía y la mastectomía. 


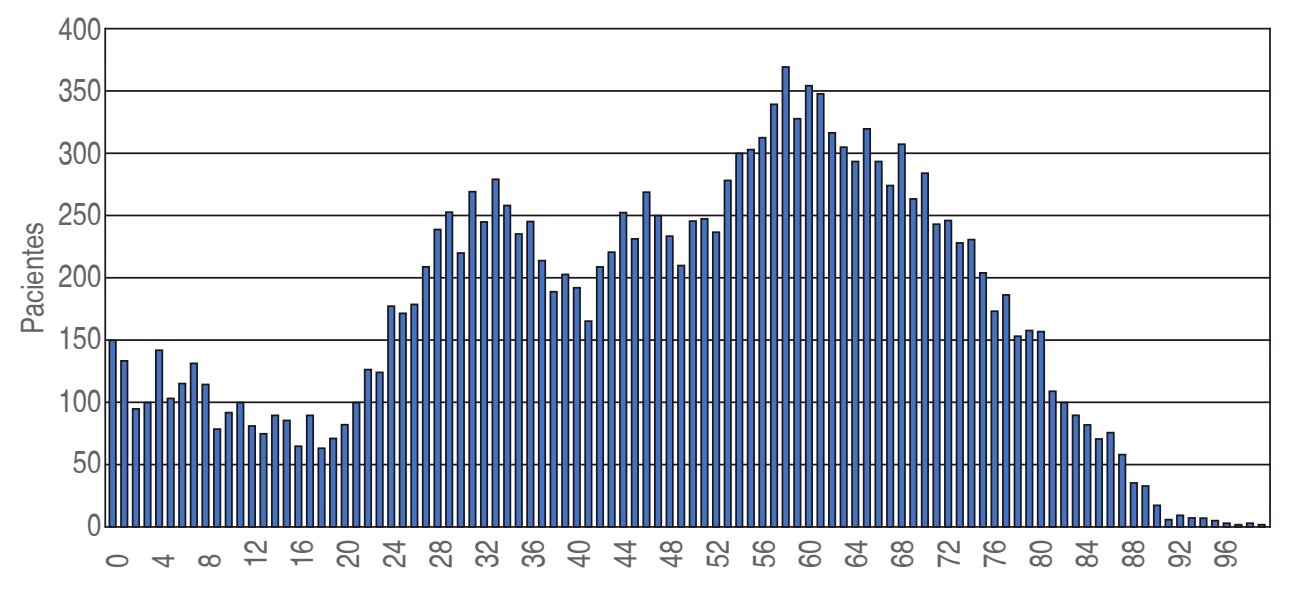

Edad
Figura 5:

Distribución por edad de los pacientes sometidos a cirugía en el CEMENAV de enero 2015 a julio 2019.
El CEMENAV, como hospital de Alto Nivel de la Secretaría de Marina, cuenta con una amplia gama de especialidades médicas y quirúrgicas al servicio de sus derechohabientes, muchas de las cuales realizan procedimientos de alta especialidad. Las especialidades que más número de procedimientos quirúrgicos tienen son cirugía general, traumatología y ortopedia, ginecología y obstetricia y oftalmología. Estas especialidades concentran la mitad de los procedimientos realizados durante el período de tiempo estudiado. Especialidades no quirúrgicas hicieron uso del quirófano para realizar procedimientos que implican sedación o anestesia, como las imágenes de resonancia o tomografía en niños, la toma de procedimientos de aspirado de médula ósea en niños y algunos adultos y endoscopía o broncoscopía.

Hablando de todos los procedimientos quirúrgicos en general, los más frecuentes fueron la facoemulsificación de catarata con colocación de lente intraocular, endoscopía, cesárea Kerr, colecistectomía laparoscópica, atención del trabajo de parto, reducción abierta y fijación interna de fracturas, apendicectomía abierta, hernioplastía inguinal, cesárea con OTB y lavado quirúrgico. Como centro de alto nivel, en el CEMENAV se realizan algunas cirugías con tecnologías muy avanzadas, como cirugía robótica, que se utilizó en 56 casos $(0.2 \%$ de todas las cirugías) para procedimientos como la histerectomía, nefrectomía, reparación de fístula vesicovaginal, colecistectomía, prostatectomía, resección anterior baja, funduplicatura, hernioplastía inguinal o cirugía por cáncer de endometrio.

\section{CONCLUSIONES}

La cirugía es una modalidad de tratamiento médico indispensable para muchas de las patologías y enfermedades como son las malformaciones, traumatismos, neoplasias, etcétera. No es posible concebir la medicina actual sin la participación de esta modalidad terapéutica. Todos los hospitales, independientemente de su nivel, tienen los servicios quirúrgicos como una prioridad. De esta manera, el número de procedimientos quirúrgicos y su complejidad irán aumentando, exigiendo que los médicos cirujanos, anestesiólogos y el personal de enfermería quirúrgica se encuentren cada vez mejor capacitados y más especializados. Asimismo, el personal que administra y dirige a los hospitales e instituciones de salud deben tener datos fidedignos y estadísticas actuales que les permitan planear estrategias para optimizar la capacidad de respuesta de los quirófanos y de los hospitales en general.

El CEMENAV es un hospital con características propias que lo vuelven único en su clase. Por un lado, es un hospital general, ya que tiene las especialidades troncales (pediatría, ginecología y obstetricia, cirugía y medicina interna) y tiene un servicio de urgencias disponible las 24 horas del día para atender padecimientos médico-quirúrgicos de estas cuatro especialidades troncales. Además, al ser el hospital de tercer nivel de la Secretaría de Marina atiende padecimientos de alta complejidad, ya que cuenta con todas las subespecialidades médicas y quirúrgicas ${ }^{(4)}$.

Lo anterior se refleja en el tipo y el número de los procedimientos quirúrgicos que se realizan en sus quirófanos y que van desde procedimientos de cirugía general, que son comunes a todos los hospitales (como apendicectomía, colecistectomía, hernioplastía, etcétera), procedimientos de atención obstétrica (trabajo de parto, cesárea, etcétera), hasta procedimientos con un alto grado de especialización (cirugía robótica, neurocirugía, cirugía oncológica, trasplantes de órganos, etcétera).

Una vez hechas las consideraciones anteriores, podemos concluir que:

1. El CEMENAV realiza en promedio 5,527 procedimientos quirúrgicos al año, con un promedio diario de 16 procedimientos.

2. Los procedimientos son constantes en todos los meses del año, con una ligera disminución en los meses de mayo y diciembre. 
3. Las cifras indican que el hospital destina los recursos de su quirófano para la atención obstétrica (atención del trabajo de parto y cesáreas con o sin OTB), la atención de padecimientos crónico-degenerativos (como la facoemulsificación de catarata con colocación de lente intraocular y las endoscopías), la atención de accidentes de traumatología (reducción de fracturas, lavados quirúrgicos de heridas, etcétera) y la resolución de padecimientos abdominales agudos como la apendicitis o colecistitis.

4. La mayor parte de los pacientes quirúrgicos son mujeres (60\%), la mayoría jóvenes (28-36 años) que se atienden por causas obstétricas.

5. La cuarta parte de los pacientes quirúrgicos fue en mayores de 65 años, con cirugías que se relacionan a padecimien- tos crónico-degenerativos (como la cirugía de catarata o las artroplastías) y neoplásicos (como la prostatectomía, histerectomía y la mastectomía por cáncer).

6. Las especialidades con más procedimientos quirúrgicos realizados son: cirugía general, traumatología y ortopedia, ginecología y obstetricia y oftalmología.

7. Procedimientos como la angioplastía coronaria, las cirugías de revascularización coronaria, las cirugías plásticas reconstructivas (principalmente de glándula mamaria), safenoablación, neurocirugía de columna lumbar y cervical, y los procedimientos de neurología intervencionista entre otros son también frecuentes y reflejan el alto grado de especialización de algunos de los procedimientos que se realizan en los quirófanos del CEMENAV.

\section{REFERENCIAS}

1. Fischler M, Cardin JL, Faucon T, Adam R. Presence of anesthesiologists and nurses in the operating room: liability of surgeons and health care facilities. J Visc Surg. 2019;156:S15-S20.

2. Lee DJ, Ding J, Guzzo TJ. Improving operating room efficiency. Curr Urol Rep. 2019;20:28.
3. Fernández-Cantón S. Otras secciones de este sitio: el IMSS en Cifras. Las intervenciones quirúrgicas. Rev Med Inst Mex Seguro Soc. 2005;43:511-520.

4. Vignolo J, Vacarezza M, Álvarez C, Sosa A. Niveles de atención, de prevención y atención primaria de la salud. Arch Med Int. 2011;33:7-11. 